\title{
CFD Simulation of the Filtration Performance of Fibrous Filter Considering Fiber Electric Potential Field
}

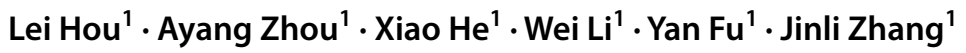

Received: 30 April 2019 / Revised: 10 May 2019 / Accepted: 16 May 2019 / Published online: 20 August 2019

(c) The Author(s) 2019

\begin{abstract}
Aiming at disclosing the quantitative effects of Coulomb forces on the filtration efficiency of aerosol particles, a threedimensional random fiber model was established to describe the microstructure of fibrous filters. Then, computational models including the flow model, particle model, and electric field model were constructed to estimate the filtration efficiency using the Fluent custom user-defined function program, neglecting the non-uniformity of the fiber potential and the particle charge distribution. The simulation results using the established models agreed with the data in the literature. In particular, the electric field force was found to be one of the important factors required to improve the filtration efficiency estimation accuracy for the ultrafine particles. Moreover, the variation tendencies of the filtration efficiency and the pressure drop of fibrous filters were studied based on the influence factors of the fiber potential, particle charge-to-mass ratio, solid volume fraction, fiber diameter, and face velocity. The established models and estimated results will provide important guidance on the design of high-efficiency particulate air filters for aerosol particles.
\end{abstract}

Keywords Filtration efficiency $\cdot$ Coulomb force $\cdot$ Three-dimensional random fiber model $\cdot$ Interception capture $\cdot$ Brownian diffusion

$\begin{array}{ll}\text { List of Symbols } \\ C_{\mathrm{C}} & \text { Cunningham correction factor } \\ d_{\mathrm{f}} & \text { Fiber diameter (m) } \\ d_{\mathrm{p}} & \text { Particle diameter (m) } \\ E & \text { Electric field strength (N/C) } \\ E_{\Sigma} & \text { Total filtration efficiency of the single fiber } \\ E_{\mathrm{R}} & \text { Interception efficiency } \\ E_{\mathrm{D}} & \text { Brownian diffusion efficiency } \\ E_{\mathrm{E}} & \text { Electrostatic force efficiency } \\ f\left(\alpha_{\mathrm{f}}\right) & \text { Dimensionless drag coefficient as a function of } \\ & \text { SVF } \\ F & \text { Dimensionless drag coefficient by the method of } \\ & \text { Happel } \\ F_{\mathrm{B}} & \text { Brown force (N) } \\ F_{\mathrm{D}} & \text { Drag (N) } \\ F_{\mathrm{E}} & \text { Electric field force (N) }\end{array}$

Electronic supplementary material The online version of this article (https://doi.org/10.1007/s12209-019-00218-7) contains supplementary material, which is available to authorized users.

Jinli Zhang

zhangjinli@tju.edu.cn

1 School of Chemical Engineering and Technology, Tianjin University, Tianjin 300350, China

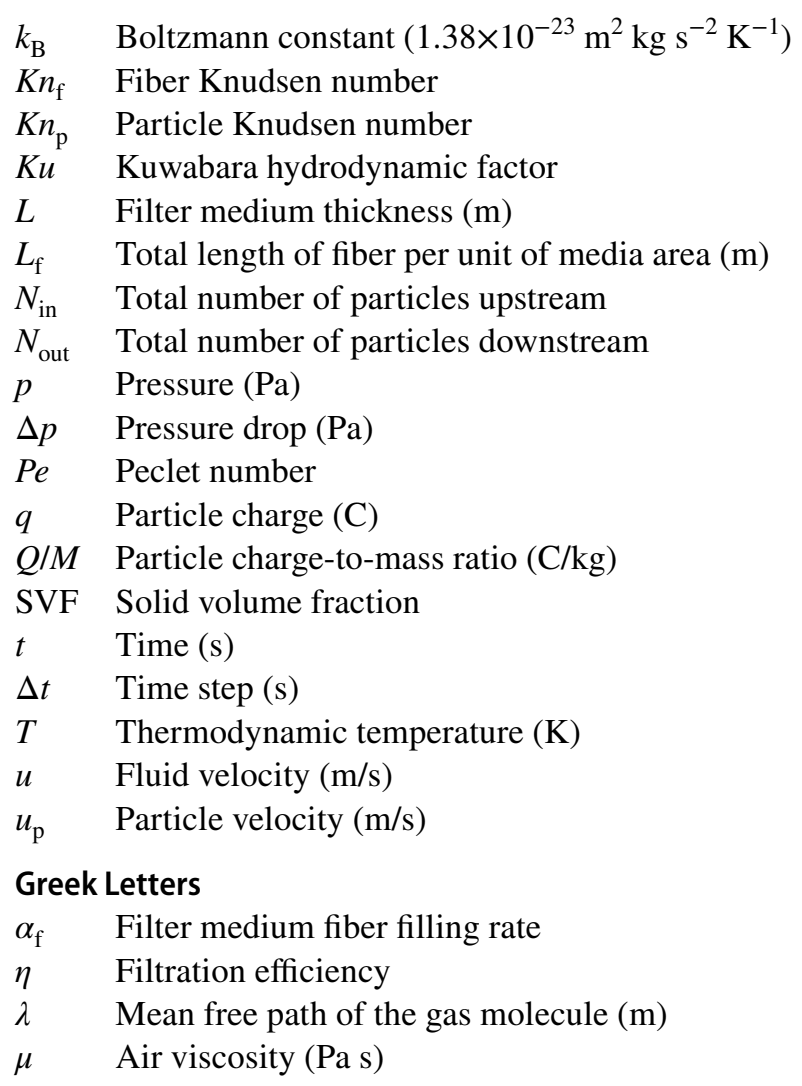




$\begin{array}{cl}\rho_{\mathrm{p}} & \text { Particle density }\left(\mathrm{kg} / \mathrm{m}^{3}\right) \\ \phi & \text { Electrostatic potential }(\mathrm{V}) \\ \xi & \text { Zero mean, unit-variance independent Gaussian } \\ & \text { random numbers }\end{array}$

\section{Introduction}

Improving the capture efficiency of fine particles has attracted more attention because of the negative impact of fine particles on human health [1-3]. In particular, highefficiency particulate air (HEPA) filters need to be designed to effectively capture ultrafine particles smaller than $300 \mathrm{~nm}$ $[4,5]$, which is important to produce clean breathable air and also to ensure safety in biosafety laboratories and the nuclear industry [6-8].

In recent years, many studies have been conducted on the synthesis of charged fibers with excellent fine particles filtration efficiency $[9,10]$. Yeom and Shim [11] added boehmite nanoparticles as the electrostatic charging agent during the electrospinning of nylon-6 (PA6) fibers and found that the charged PA6 fibers provided a filtration efficiency of $27.8 \%$ toward $300 \mathrm{~nm}$ aerosol, which was much higher than that of uncharged fibers $(6.3 \%)$, without changing the resistance. Tang et al. [12] studied the filtration efficiency of an electret filter for 3-500 nm particles and concluded that the collection efficiency of charged particles $(50-100 \mathrm{~nm})$ increased by over $20 \%$. However, how to estimate the quantitative effect of electrostatic interaction on improving the filtration efficiency of fine particles remains unclear.

According to the classical filtration theory, the filtration performance of fibers in a stable phase is associated with five capture mechanisms: interception effect, inertial collision, Brownian diffusion, electrostatic interaction, and gravity [13-16]. Previous studies have reported numerical simulations of filtration performance considering Brownian diffusion, interception, and inertial impaction [17-21]. For example, a single fiber model has been established to simulate the collection efficiency, neglecting the interactions among the fibers [22-24]. Moreover, a parallel and staggered multi-fiber filtration model has been developed to simulate the collection efficiency [25]. As for the effect of electrostatic interactions on the collection efficiency, Wang [26] presented a comprehensive review of the theoretical and experimental studies of electrostatic forces in filtration. However, thus far, no report on the numerical simulation with electrostatic forces has been found in the literature.

Considering the three-dimensional (3D) porous microstructure of fibrous filters, in this study, we developed a software-coupling method, interconnecting MATLAB, AutoCAD, ICEM, and Fluent software, to generate a 3D random fiber model that approximately describes the microstructure of fibrous filters. Afterward, computational models considering fiber electric potential field were developed using the Fluent custom user-defined function (UDF) program, assuming a uniform fiber potential and neglecting the non-uniformity of the particles charge distribution. After the model verification, numerical simulations were performed to further clarify the regulations on improving the filtration efficiency of fibrous filters, which will provide important guidance on the design of HEPA filters.

\section{Generating Three-Dimensional Random Fiber Model}

Shou et al. [27] suggested that a random fiber media model would provide more permeability, compared with the ordered fiber arrays. In this study, assuming a random fiber structure in the filter, we developed a coupling method, involving MATLAB R2014a (https://www.mathworks.cn), AutoCAD 2017 (https://www.autodesk.com.cn), ICEM 15.0 (https://www.ansys.com), and Fluent 15.0 (https://www. ansys.com/Products/Fluids), to generate a 3D random fiber model that approximately describes the porous microstructure of fibrous filters.

The first step was to generate the pillar model mimicking the filter fiber by coupling MATLAB and AutoCAD. We wrote a program via a randomized algorithm in MATLAB to generate a random point matrix. Then, the random point matrix was inputted into AutoCAD to form a set of line segments, and consequently, the pillar models were constructed with an average diameter on the basis of the centered line segments. For each line segment, the two terminal points should have the same $x$ coordinate, to maintain all the fibers located horizontally.

Second, the geometries of the pillar models were inputted into ICEM to generate the mesh simulation of the porous microstructure of fibrous filters, and the corresponding grid file was inputted into Fluent as the 3D random fiber model for further simulation. Figure 1 shows the 3D random fiber model generated by the above software-coupling method, with a solid volume fraction (SVF) of 3\%, thickness of $1 \mu \mathrm{m}$, and fiber diameter $\left(d_{\mathrm{f}}\right)$ of $370 \mathrm{~nm}$. The inlet and outlet of the calculation zone are assumed to be in the undisturbed flow field and placed, respectively, at distances of seven times $d_{\mathrm{f}}$ upstream and downstream of the filter medium.

The boundary should be set far away from the porous medium to avoid interference with the flow field. Hosseini and Tafreshi [21] performed an air filtration simulation, with the inlet and the outlet of the calculation zone, respectively, at distances of 20 times $d_{\mathrm{f}}$ and five times $d_{\mathrm{f}}$ upstream and downstream of the filter medium. Babaie et al. [28] set the length of the inlet and outlet media to 7 and 5 times the fiber diameter, respectively. In our work, the inlet and the outlet were first set at distances of 20 times $d_{\mathrm{f}}$ upstream and 


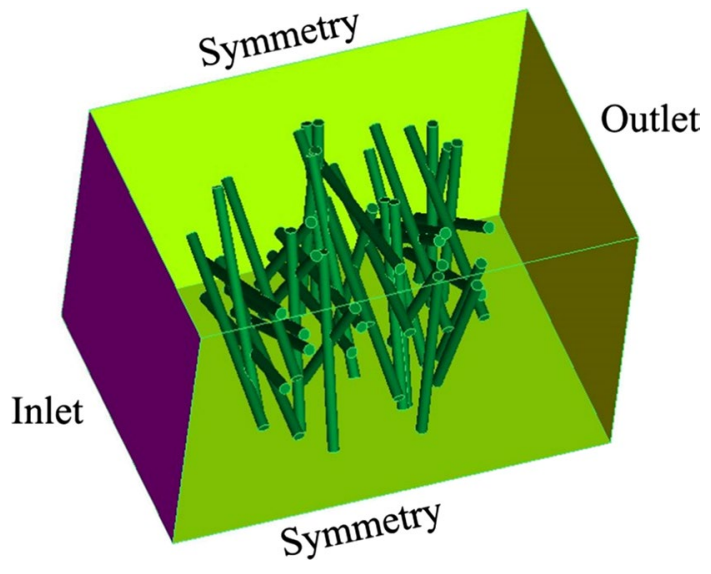

Fig. $13 \mathrm{D}$ random fiber model with SVF of 3\%, thickness of $1 \mu \mathrm{m}$, and fiber diameter of $370 \mathrm{~nm}$

downstream of the simulation medium, and the corresponding velocity contour is shown in Fig. 2a. The distance was reduced to seven times $d_{\mathrm{f}}$ upstream and downstream of the medium, and the velocity contour displayed in Fig. $2 \mathrm{~b}$ was obtained. The velocity flow field in Fig. $2 b$ is similar to that obtained with the large distance of 20 times $d_{\mathrm{f}}$ (Fig. 2a). Therefore, we adopted the distance of seven times $d_{\mathrm{f}}$ in the simulation to save the computer's running space.

\section{Computational Models and Simulation Method}

\section{Flow Model}

In the fiber medium, the Reynolds number was small, and thus, the inertial force was much smaller than the viscous force. The viscous force was dominant, while the inertial force was neglected, and the air followed the Stokes flow [29]. The finite volume method implemented by Fluent code was used to solve the gas flow field. The continuity equation and momentum equation of fluid flow are as follows:
Fig. 2 Velocity contour of flow field (SVF of 4\%, thickness of $10 \mu \mathrm{m}$, and fiber diameter of $370 \mathrm{~nm}$ ) with boundary distances of $20 d_{\mathrm{f}}(\mathbf{a})$ and $7 d_{\mathrm{f}}(\mathbf{b})$

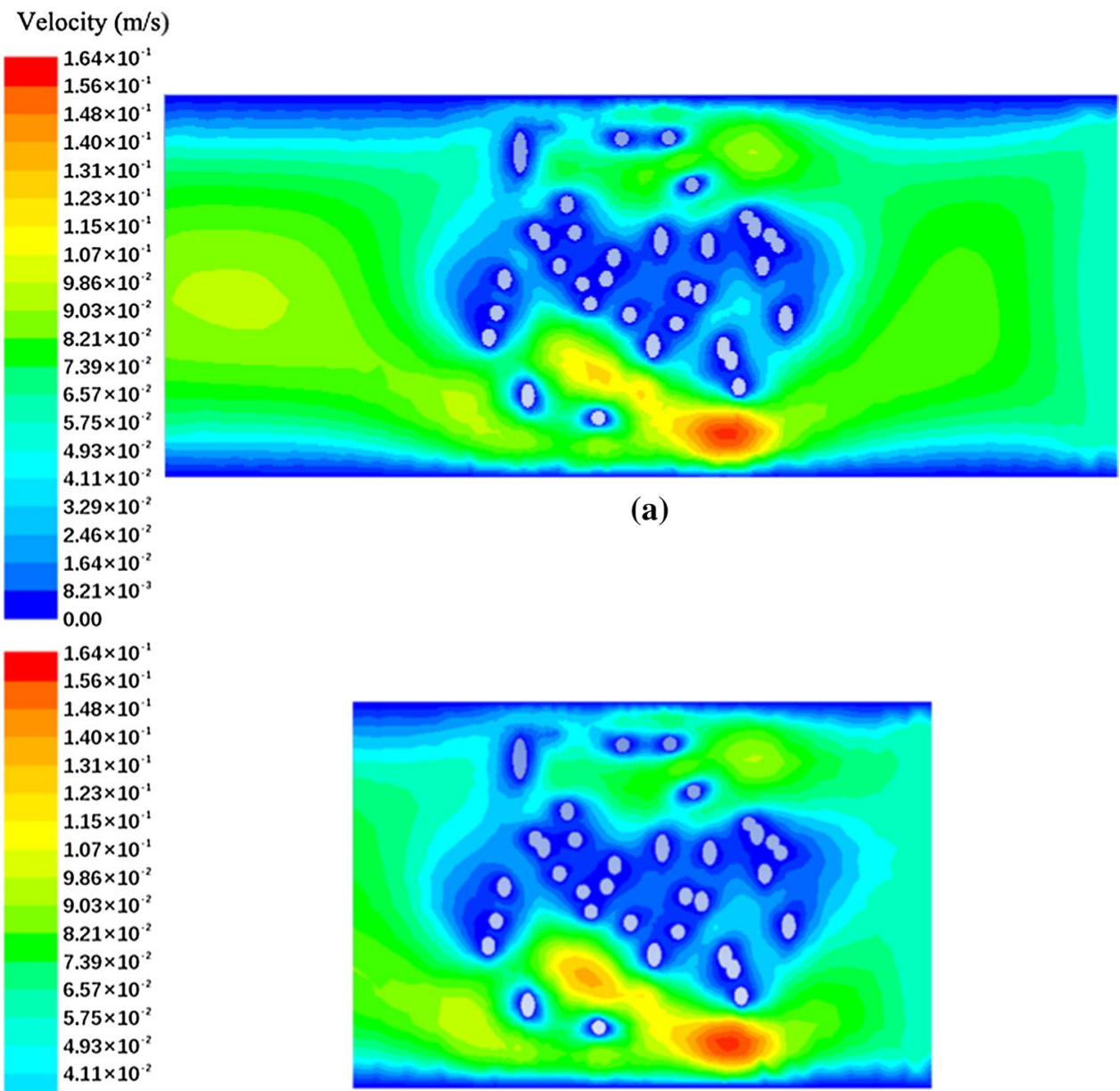

(b) 
$\nabla \boldsymbol{u}=0$

$\nabla \boldsymbol{p}=\mu \nabla^{2} \boldsymbol{u}$

where $\boldsymbol{u}$ is the fluid velocity $(\mathrm{m} / \mathrm{s}) ; \boldsymbol{p}$ is the pressure; and $\mu$ is air viscosity (Pa s). As shown in Fig. 1, the entrance of the computational domain was set as a velocity inlet, and a pressure boundary condition was applied to the outlet. The sides of the domain were set as symmetric boundary conditions.

\section{Particle Model}

In Fluent, the Euler method and the Lagrangian method are generally used to simulate the gas-solid two-phase flow process, whereby the Euler method is applied to a mixture with a higher dispersion volume fraction, while the Lagrangian method has a good simulation effect for a mixture with a dilute dispersed phase. Here we adopted the Lagrangian method to simulate the fiber filtration process.

In the Lagrangian method, the particle position is obtained by integrating the force balance equation of the particle:

$\frac{\mathrm{d} u_{\mathrm{p}}}{\mathrm{d} t}=F_{\mathrm{D}}+F_{\mathrm{B}}+F_{\mathrm{E}}=\frac{18 \mu}{d_{\mathrm{p}}^{2} \rho_{\mathrm{p}} C_{\mathrm{C}}}\left(u-u_{\mathrm{p}}\right)+\xi \sqrt{\frac{216 \mu k_{\mathrm{B}} T}{\pi \rho_{\mathrm{p}}^{2} d_{\mathrm{p}}^{5} \Delta t}}+q E$

where $F_{\mathrm{D}}, F_{\mathrm{B}}$, and $F_{\mathrm{E}}$ represent the drag, Brown force, and electric field force $(\mathrm{N})$, respectively; $u_{\mathrm{p}}$ is the particle velocity $(\mathrm{m} / \mathrm{s}) ; C_{\mathrm{C}}$ is the Cunningham correction factor that is calculated via $C_{\mathrm{C}}=1+K n_{\mathrm{p}}\left(1.257+0.4 \mathrm{e}^{-1.1 / K n}{ }_{\mathrm{p}}\right)[21,30,31]$; $d_{\mathrm{p}}$ is the particle diameter $(\mu \mathrm{m}) ; K n_{\mathrm{p}}=\lambda / d_{\mathrm{p}}$ is the Knudsen number; $\lambda$ is the mean free path of the gas molecule $(\mathrm{m}) ; \rho_{\mathrm{p}}$ is particle density $\left(\mathrm{kg} / \mathrm{m}^{3}\right.$, here $1000 \mathrm{~kg} / \mathrm{m}^{3}$ is adopted); $\xi$ represents a random number in a standard normal distribution with a zero mean and a variance of $1 ; k_{\mathrm{B}}$ is the Boltzmann constant; $T$ is the thermodynamic temperature $(\mathrm{K}) ; \Delta t$ is the time step (s); $q$ is the particle charge (C); and $E$ is the electric field strength (N/C).

The discrete phase model (DPM) in Fluent was used to solve the force balance equation of the particle. The boundary conditions of the calculation domain inlet and outlet were set as the "escape"; the boundary condition of the fiber surface was set as the "trap"; and the boundary condition of the wall was set as the "reflect". The unidirectional coupling method was used to deal with the interaction force between air and particles, ignoring the interaction force among the particles. The standard DPM model treats particles as mass points and only considers the trapping of particles; however, it neglects the effect of inertia on nanoparticles. In air filtration, the main factors that determine the capture efficiency are the interception effect and the diffusion effect. Therefore, the UDF was programmed to improve the DPM model, and the distance between the particle center trajectory and the fiber surface was monitored in real time to introduce the interception effect on the particle. As this distance was shorter than the particle radius, the interception effect was significant to capture the particle. In addition, the Brownian force numerical formula [21] was introduced by the UDF to calculate the influence of the Brownian diffusion motion on the particle interception effect.

The present work is associated with the treatment of indoor air in biosafety laboratories containing a low concentration of ultrafine particles, whereby a total removal of the ultrafine particles is required. During the simulation, the change in geometry of the domain was neglected as the particles were captured by the fibers; that is, if the particles were captured by the fibers, they would be deleted from the domain. Such an assumption has also been adopted in previous studies. Hosseini and Tafreshi [32] established a model in disordered 2D domains for the filtration of particles smaller than $500 \mathrm{~nm}$, assuming that the particles captured by the fibers would be removed from the domain. Jin et al. [33] modeled the filtration of particles larger than $500 \mathrm{~nm}$, assuming that the particles would be removed from the domain when they were captured by the fiber.

Figure 3 displays a typical standardized particle concentration contour. The normalized concentration of particles adjacent to the fiber was zero, indicating that the particles were deposited on the fiber, and the normalized concentration at the outlet was substantially zero, indicating that the particles were intercepted by the filter media.

\section{Electric Field Model}

Electrostatic forces occur when particles and fibers are charged or an external electric field is applied to the medium. In theory, the electrostatic forces include Coulomb force, image force,

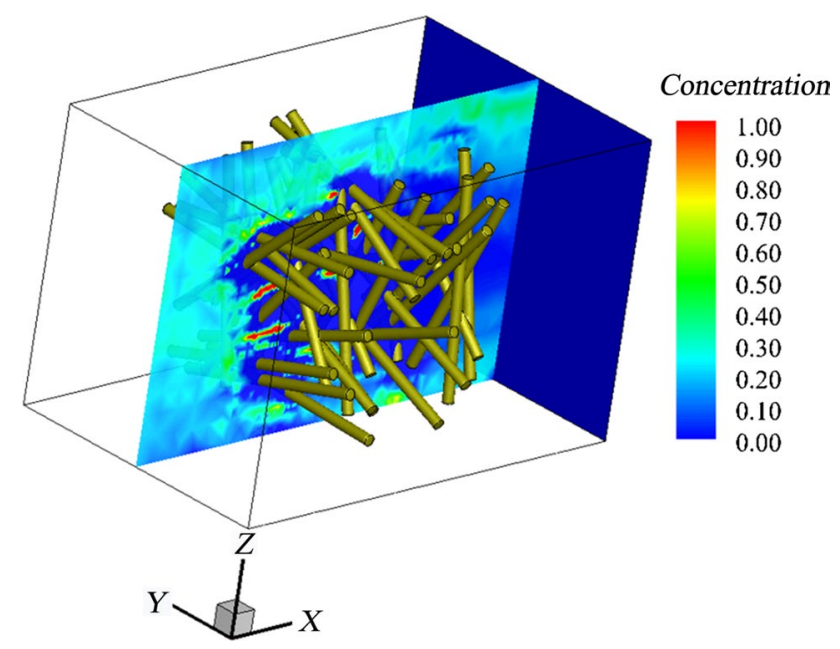

Fig. 3 Typical standardized particle concentration contour, with $d_{\mathrm{p}}=300 \mathrm{~nm}, d_{\mathrm{f}}=370 \mathrm{~nm}$, and $u=0.05 \mathrm{~m} / \mathrm{s}$ 
and polarization force; however, in the literature, each force is usually considered according to proper assumptions. For instance, Nielsen and Hill [34] studied the collection efficiency of particles by a single spherical collector in wet scrubbers, considering the Coulomb forces, electrical image forces, and external electric field force. They concluded that the effect of the charged-particle and charged-collector image forces on the particle trajectory was much smaller than that of Coulomb forces. D'Addio et al. [35] established a particle scavenging model that considered Coulomb forces in the wet scrubbing of submicron particles (100-450 nm) and successfully described the scavenging coefficient of wet electrostatic scrubbing with charged particles and droplets. Hamaguchi and Farouki [36] estimated the magnitude of the polarization force under typical glow discharge conditions and high particulate density. Kanaoka et al. [37] simulated the agglomerative deposition process of fine aerosol particles $(390 \mathrm{~nm})$ under a dust-laden condition and concluded that the Coulomb force essentially determined the trajectory of charged particles. Zuo et al. [38] studied the removal of particulate matter in polluted air, quantitatively analyzed the forces exerted on the moving particles $(10 \mu \mathrm{m})$, and concluded that the image force could be neglected, compared with the Coulomb force. Therefore, we focused on the Coulomb force to study the effect of electrostatic force on the filtration efficiency for low-concentrated ultrafine particles.

It has been suggested that the electric field is determined only by the fiber potential when the particle charge is small [39]. As for the particle charge distribution, Hoppel [40, 41] studied the charge distribution of aerosols using ion-aerosol balance equations and concluded that the Boltzmann statistics could provide an accurate estimation of the charge distribution for aerosol particles larger than $0.5 \mu \mathrm{m}$, whereas for smaller particles, the accuracy was reduced. Dhanorkar and Kamra [42] studied the particle charge distribution in a polluted atmosphere with a total aerosol concentration of 1000 particles $/ \mathrm{cm}^{3}$ and found that the particle charge distribution depended on the aerosol concentration and ionization rate. In the present work, we focused on the removal of ultrafine particles with low concentration in the atmosphere of biosafety laboratories. It is reasonable to neglect the charge distribution of ultrafine particles and assume that the charged particles behavior does not affect the fiber potential field.

The fiber electrostatic potential field was established by writing the user-defined scalar (UDS). A UDS was defined to represent the potential field, and three user-defined memory (UDM) locations were specified to store the field strength in $x, y$, and $z$ directions. The force acting on the particle by the electric field was proportional to the spatial gradient of the electrostatic potential, and the Laplace equation (UDF) of the electrostatic potential is expressed as Eq. (4).

$$
\frac{\partial^{2} \phi}{\partial x^{2}}+\frac{\partial^{2} \phi}{\partial y^{2}}+\frac{\partial^{2} \phi}{\partial z^{2}}=0
$$

where $\phi$ denotes electrostatic potential.

The electric field $E$ (UDF) generated by the electrostatic potential is calculated using Eq. (5).

$E=-\nabla \phi$

The force exerted by the potential field on the particles is defined as

$F=q E$

where $q$ denotes the amount of charge of the particles.

The zeta potential of Whatman EPM 2000 glass fibers was measured at different $\mathrm{pH}$ environments, as shown in Fig. $\mathrm{S} 1$ in the supporting information. Assuming that the surface potentials of the fibers were uniform, the fiber potential was set as $-0.3 \mathrm{~V}$, which corresponds to the value at neutral $\mathrm{pH}$.

The potential values on the inlet and the outlet of the calculation domain were set as zero; the potentials on the other boundaries of the calculation domain were also set as zero; and the fiber potential was set as $-0.3 \mathrm{~V}$, which corresponds to the zeta potential measured at $\mathrm{pH}$ 7.0.

D'Addio et al. [35] measured the charge of an aerosol with a particle size distribution of $100-450 \mathrm{~nm}$ and reported a particle charge-to-mass ratio $(Q / M)$ of $0.075-0.1 \mathrm{C} / \mathrm{kg}$. In the present study, neglecting the non-uniformities of the charge distribution of the particles, the particle $Q / M$ ratio was set as $0.085 \mathrm{C} / \mathrm{kg}$. Since the fiber surface was electrically identical to the particles, an electrostatic repulsion force was generated to cause the particle trajectory to wrap around, so that a portion of the particles was confined to the filter medium region by the interception.

The UDS contour represents the distribution of the potential field. The model overall potential contour of the constructed electric field is shown in Fig. S2. Since the potential of $-0.3 \mathrm{~V}$ was applied only to the fiber surface in the entire calculation domain, it can be seen that the intermediate filter medium region had a lower overall potential value.

The UDM contour illustrates the model field strength distribution of the constructed electric field, as shown in Fig. S3. The field strength is defined by the negative gradient of the potential, indicating that the faster the potential drops, the larger the gradient and the stronger the field strength. In the filter medium region, the potential value of the wall was 0 on both sides, and the potential value applied to the surface of the fiber was $-0.3 \mathrm{~V}$; thus, the potential gradient on both sides of the filter medium was extremely large, whereas it was not strong enough in the intermediate fiber aggregation area.

Figure 4 compares the particle motion trajectory curves in the presence of a potential field with those in the absence of a potential field. Under the conditions of $u=0.05 \mathrm{~m} / \mathrm{s}$ and $d_{\mathrm{p}}=300 \mathrm{~nm}$, without a potential field, most of the particles could not be captured by the fiber surface (Fig. 4a). However, in the presence of a potential field, a large number of 
(a)

Velocity $(\mathrm{m} / \mathrm{s})$

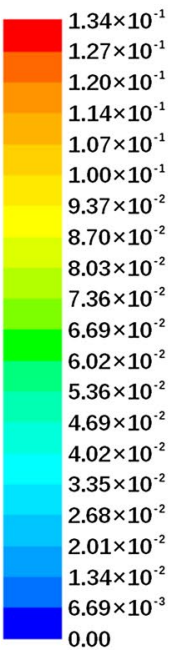

(b)

Velocity $(\mathrm{m} / \mathrm{s})$

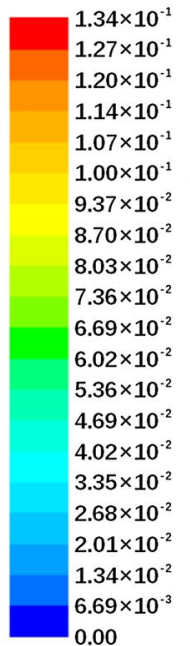

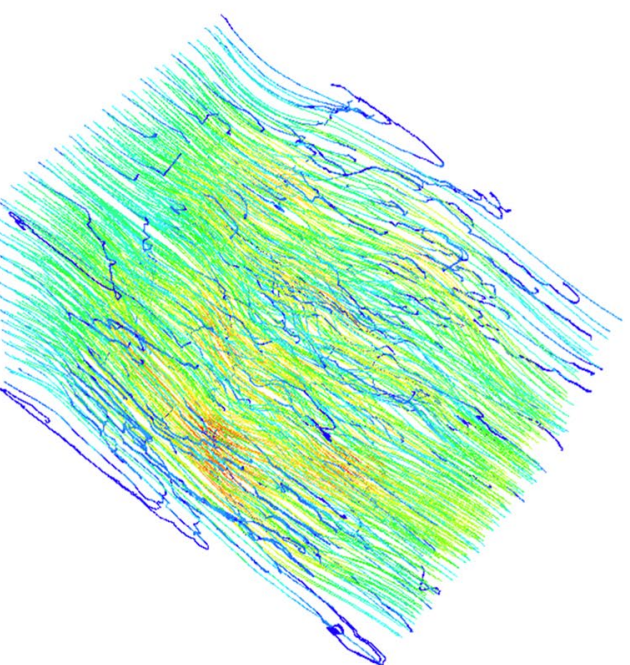

Fig. 4 Motion trajectory curves of the particles: $\mathbf{a}$ in the absence of potential field; $\mathbf{b}$ in the presence of potential field

Table 1 Effect of Coulomb force on the retention ratio (fiber potential $=-0.3 \mathrm{~V}, Q / M$ value $=-0.085 \mathrm{C} / \mathrm{kg}, u=0.05 \mathrm{~m} / \mathrm{s}$, and $\left.d_{\mathrm{p}}=300 \mathrm{~nm}\right)$

\begin{tabular}{llll}
\hline & $N_{\text {in }}$ & $N_{\text {out }}$ & $\left(N_{\text {in }}-N_{\text {out }}\right) / N_{\text {in }}$ \\
\hline Without Coulomb force & 431 & 430 & 0.0023 \\
With Coulomb force & 431 & 264 & 0.388 \\
\hline
\end{tabular}

particles changed the motion trajectory due to the electrostatic force and were captured by the fiber surface (Fig. 4b). In addition, we compared the retention ratio $\left[\left(N_{\text {in }}-N_{\text {out }}\right) / N_{\text {in }}\right]$ with and without considering the Coulomb force for the $300 \mathrm{~nm}$ particles, where $N_{\text {in }}$ and $N_{\text {out }}$ are the total numbers of particles at the inlet and outlet, respectively. As listed in Table 1, without considering the electrostatic force, the retention ratio was 0.0023 , which is much lower than that when the electrostatic force was considered (0.388). This confirms that the Coulomb force plays an important role in the filtration efficiency.

\section{Mesh Independence Test}

In the Fluent simulation calculation, the mesh density has a great influence on the numerical calculation results. Generally, the denser the mesh is, the more accurate the numerical results. Therefore, the numerical simulation results have practical significance only when the increase in the number of grids has little effect on the calculation results. We studied the relationship between the simulation results and the mesh density by increasing the number of grid points around the circular cross section of the fiber. As the number of grids around the cross section of the fiber increased, the pressure

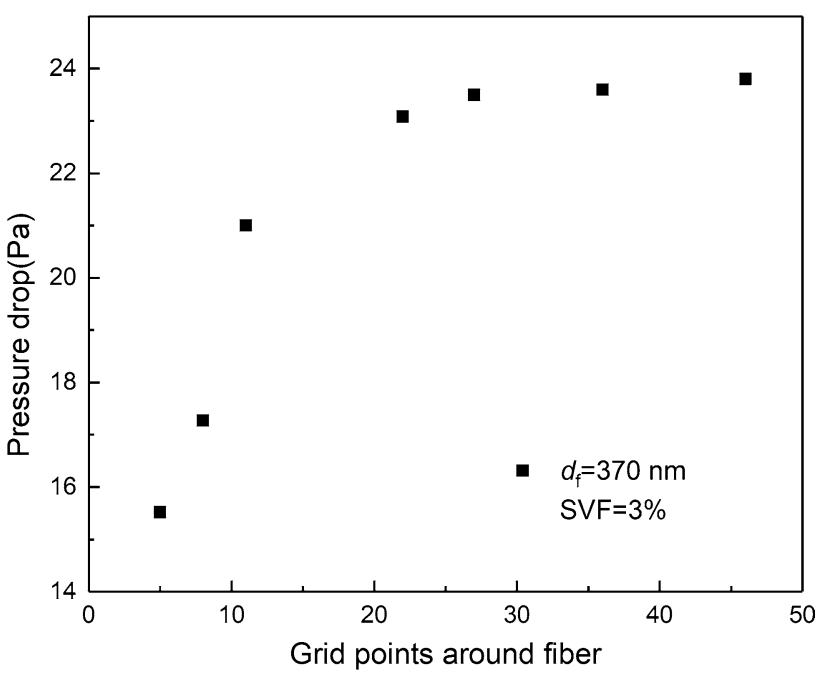

Fig. 5 Relationship between pressure drop and the number of grid points around the fiber

drop eventually reached a platform (Fig. 5). When the number of grid points around a single fiber was 46 , the difference between the pressure drop data was negligible, compared with that when the number was 27 . Thus, the number of grid points was set as 27 to ensure that the numerical simulation results were independent of the mesh density.

\section{Model Verification}

First, we performed the steady-state calculation of the single gas-phase flow in the laminar flow state. After the calculation results converged, the stable air flow field was obtained, 
and then the inert particles with a density of $1050 \mathrm{~kg} / \mathrm{m}^{3}$ were introduced through a DPM. The presence of the particle phase caused the perturbation of the continuous field, and the motion trajectory of each particle was tracked by the Lagrangian method, and their positions were monitored to perform the operation of the gas-solid two-phase flow field.

\section{Pressure Drop}

Pressure drop is an important property of fibrous filters. Kuwabara [29] first derived a formula to estimate the pressure drop across fibrous filters. Since then, a series of empirical equations have also been developed to estimate the pressure drop using parameters involving the fiber media thickness, SVF, air viscosity, gas velocity, and fiber diameter $[43,44]$. In the present study, we compared our simulation results with those of two popular models, by Davies [45] and Happel [46].

Equations (7) and (8) show the correlation of Davies' model [45] to calculate the pressure drop of a fiber medium.

$\frac{\Delta p}{L}=f\left(\alpha_{\mathrm{f}}\right) \frac{\mu u}{d_{\mathrm{f}}^{2}}$

$f\left(\alpha_{\mathrm{f}}\right)=64 \alpha_{\mathrm{f}}^{\frac{3}{2}}\left(1+56 \alpha_{\mathrm{f}}^{3}\right)$

where $\Delta p$ is the pressure drop; $\alpha_{\mathrm{f}}$ is the filter medium fiber filling rate; $L$ is the filter medium thickness; and $f\left(\alpha_{\mathrm{f}}\right)$ is the dimensionless drag coefficient as a function of SVF.

Pressure drop can also be calculated using the Darcy equation [47]:

$\Delta p=\mu u L_{\mathrm{f}} F$

where $L_{\mathrm{f}}$ is the total length of fiber per unit of media area, calculated as follows:

$L_{\mathrm{f}}=\frac{4 \alpha_{\mathrm{f}} L}{\pi d_{\mathrm{f}}^{2}}$

Combining the above two equations, Eq. (11) is obtained.

$\Delta p=\frac{4 \mu \alpha_{\mathrm{f}} u L}{\pi d_{\mathrm{f}}^{2}} F$

Equation (12) is the modeling equation to estimate the dimensionless drag coefficient $F$ by Happel's method [46].

$F=\frac{8 \pi}{-\ln \alpha_{\mathrm{f}}-\left(1-\alpha_{\mathrm{f}}^{2}\right) /\left(1+\alpha_{\mathrm{f}}^{2}\right)}$

Figure 6 shows the estimated pressure drop under various face velocities using different modeling equations, compared with the experimental data in Ref. [4]. The results simulated

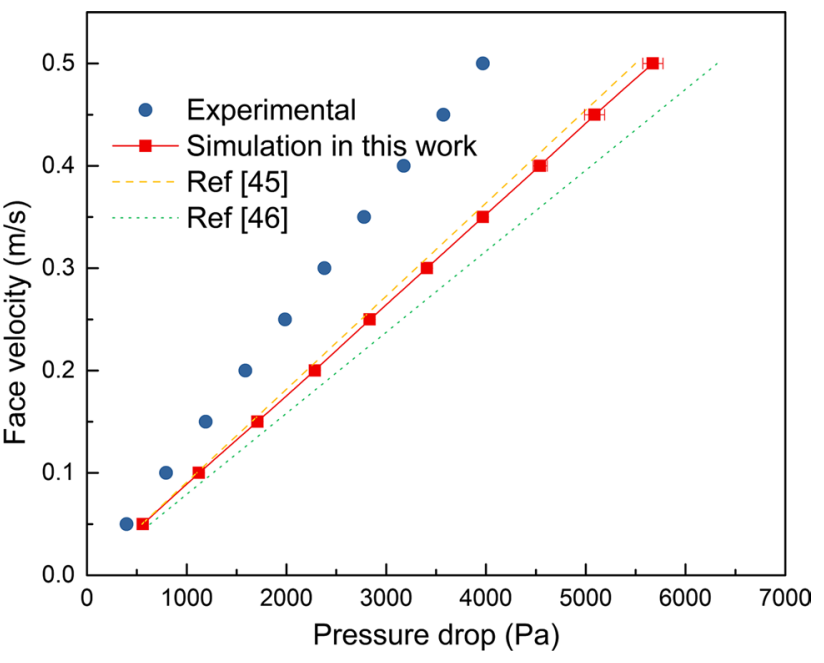

Fig. 6 Estimated pressure drop results as a function of face velocity, and the experimental data from Ref. [4]

in this work are close to those calculated by Davies' model [45]. At the face velocity lower than $0.5 \mathrm{~m} / \mathrm{s}$, the relative deviation between the simulated pressure drop and the experimental data is about $40 \%$. For example, when the velocity is $0.05 \mathrm{~m} / \mathrm{s}$, the experimental value of pressure drop is $397 \mathrm{~Pa}$, the simulated value is $559 \mathrm{~Pa}$, and the calculation yield is $40.8 \%$. The large deviation is probably due to the fibers orientations changing from in-plane to through-plane [48]. In addition, the assumption of uniform fiber diameter in the model will result in some deviation since the fiber diameter in the actual filter media has a range of distribution.

\section{Filtration Efficiency}

The filtration efficiency of the fibrous filter media is defined as

$\eta=1-\frac{N_{\text {out }}}{N_{\text {in }}}$

where $N_{\text {in }}$ and $N_{\text {out }}$ are the total numbers of particles at the inlet and outlet, respectively.

The filtration efficiency can be calculated by the fiber structural parameters, and the initial filtration efficiency $(\eta)$ is estimated using Eq. (14) [49].

$\eta=1-\exp \left[\frac{-4 \alpha_{\mathrm{f}} E_{\Sigma} L}{\pi d_{\mathrm{f}}\left(1-\alpha_{\mathrm{f}}\right)}\right]$

where $E_{\Sigma}$ is the total filtration efficiency of the single fiber model.

The general single fiber capture efficiency is associated with four retention mechanisms: the interception, inertial 
impaction, Brownian diffusion, and electric field force. For low-speed submicron particles, the relatively small inertial impaction is usually negligible; therefore, the total filtration efficiency of the single fiber model is calculated using Eq. (15) [50].

$E_{\Sigma}=1-\left(1-E_{\mathrm{R}}\right)\left(1-E_{\mathrm{D}}\right)\left(1-E_{\mathrm{E}}\right)$

where $E_{\mathrm{R}}, E_{\mathrm{D}}$, and $E_{\mathrm{E}}$ are the capture efficiencies due to the interception effect, Brownian diffusion, and electric field force, respectively.

At lower flow rates, the diffusion and Coulomb forces synergistically capture the particles due to a longer residence time. As the gas flow rate increases, the contribution of the diffusion mechanism to the media filtration efficiency becomes smaller, and the speed-independent interception mechanism becomes dominant.

The thermal motion of gas molecules collides with the particles to produce Brownian motion of the particles, which is more pronounced for small-diameter particles. Table 2 presents several reported modeling equations for fiber capture efficiency induced by Brownian diffusion, considering different parameters, such as particle size and fiber radius. Lee and Liu [51] adopted the parameters of volume fraction of filter, fiber radius, and flow velocity in the modeling equation to calculate $E_{\mathrm{D}}$. On the basis of the above parameters, including a fiber diameter of $370 \mathrm{~nm}$ and SVF of $3 \%$ for particles 10-500 nm, the Brownian force efficiency was calculated accordingly using the equations in Table 2 , and the results are displayed in Fig. 7, together with the simulated results in this work. The $E_{\mathrm{D}}$ profile simulated in this work approximates that calculated by Stechkina and Fuchs's formula [52] with a particle size of 10-400 nm, and it was close to that calculated by Liu and Rubow's formula [53] when the particle size was $400-500 \mathrm{~nm}$.

Table 3 presents some modeling equations in the literature to calculate the capture efficiency $\left(E_{\mathrm{R}}\right)$ caused by the interception effect. We also calculated the $E_{\mathrm{R}}$ value, with a

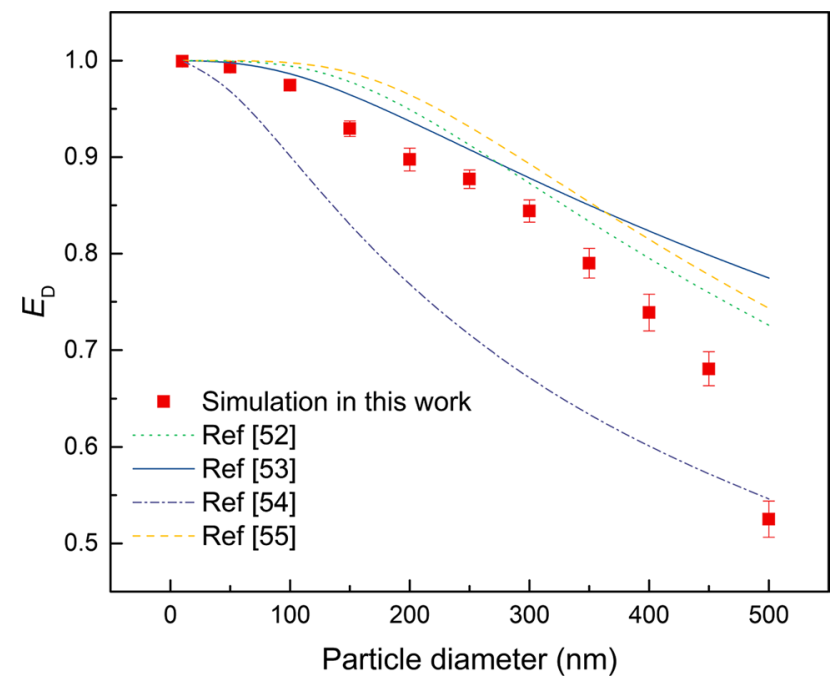

Fig. $7 E_{\mathrm{D}}$ profile simulated in this work compared with those calculated by other models (fiber diameter $=370 \mathrm{~nm} ; \mathrm{SVF}=3 \%$ )

fiber diameter of $370 \mathrm{~nm}$ and SVF of 3\%, for the $10-500 \mathrm{~nm}$ particles according to these equations and plotted $E_{\mathrm{R}}$ profiles (Fig. 8) to compare with the $E_{\mathrm{R}}$ profile simulated in this work. The simulated $E_{\mathrm{R}}$ profile approximates that calculated by the formula proposed by Pich [56] as the particle size was in the range of $10-200 \mathrm{~nm}$, while for large particles (200-500 nm), our simulated results are close to that calculated by the formula proposed by Lee and Gieseke [57].

Adopting the experimental data from Ref. [4], we simulated the filtration efficiency for the $10-500 \mathrm{~nm}$ particles, considering the filtration caused by the Coulomb force. Bortolassi et al. [4] utilized an HEPA-2 filter made by glass fiber (EPM2000), without investigating the value of the fiber potential. However, Oyama et al. [58] reported that the glass fiber sheet generally had a negative charge, which enabled the separation of the bound/free antigen/antibody. Therefore, we selected $-0.3 \mathrm{~V}$ as the default potential of the fiber surface, based on the above simulation. As a control,
Table 2 Equations to calculate the Brownian-capture efficiency $\left(E_{\mathrm{D}}\right)$

\begin{tabular}{|c|c|c|}
\hline Expression & Remark & Reference \\
\hline$E_{\mathrm{D}}=2.9 K u^{-\frac{1}{3}} P e^{-\frac{2}{3}}+0.62 P e^{-1}$ & $\begin{array}{l}P e \gg 1 \\
\text { Analysis of boundary layer }\end{array}$ & Stechkina and Fuchs [52] \\
\hline$E_{\mathrm{D}}=1.6\left(\frac{1-\alpha}{K u}\right)^{\frac{1}{3}} P e^{-\frac{2}{3}} C_{\mathrm{d}}$ & $\begin{array}{l}C_{\mathrm{d}}=1+0.388 K n_{f}\left(\frac{(1-\alpha) P e}{\mathrm{Ku}}\right)^{\frac{1}{3}} \\
10^{-2}<K n_{\mathrm{f}}<10^{-1} \\
\text { Slip flow regime }\end{array}$ & Liu and Rubow [53] \\
\hline$E_{\mathrm{D}}=1.6\left(\frac{1-\alpha}{K u}\right)^{\frac{1}{3}} P e^{-\frac{2}{3}} C_{\mathrm{d}} C_{\mathrm{d}}^{\prime}$ & $\begin{array}{l}C_{\mathrm{d}}^{\prime}=\frac{1}{1+\left(E_{\mathrm{D}}\right)_{\text {Liu and Rubow }(1990)}} \\
10^{-2}<K n_{\mathrm{f}}<10^{-1} \\
0.02 \mu \mathrm{m}<d_{\mathrm{p}}<0.5 \mu \mathrm{m}\end{array}$ & Payet et al. [54] \\
\hline$E_{D}=1.6\left(\frac{1-\alpha}{K u}\right)^{\frac{1}{3}} P e^{-\frac{2}{3}}$ & $\begin{array}{l}0.029<\alpha_{\mathrm{f}}<0.1 \\
\text { Theoretical model }\end{array}$ & Lee and Liu [55] \\
\hline
\end{tabular}


Table 3 Equations to calculate the interception capture efficiency $\left(E_{\mathrm{R}}\right)$

\begin{tabular}{llll}
\hline Expression & Remark & References \\
\hline$E_{\mathrm{R}}=0.6 \frac{1-\alpha}{K u} \frac{R^{2}}{1+R}$ & $(20)$ & $0.01 \mathrm{~m} / \mathrm{s}<u<0.30 \mathrm{~m} / \mathrm{s}$ & Lee and Liu [55] \\
& & $0.05 \mu \mathrm{m}<d_{\mathrm{p}}<1.3 \mu \mathrm{m}$ & \\
& & $0.0086<\alpha_{\mathrm{f}}<0.151$ & \\
& & In good agreement for particles & \\
& & $d_{\mathrm{p}}<0.5 \mu \mathrm{m}$ & \\
& & No slip flow at gas-fiber interface & Pich [56] \\
$E_{\mathrm{R}}=\frac{(1+R)^{-1}-(1+R)+2(1+1.996 K n)(1+R) \ln (1+R)}{2\left(-0.75-0.5 \ln \alpha_{\mathrm{f}}\right)+1.996 K n\left(-0.5-\ln \alpha_{\mathrm{f}}\right)}$ & $(21)$ & In good agreement for particles & \\
& & $d_{\mathrm{p}}>20 \mathrm{~nm}$ & Lee and Gieseke [57] \\
& & $m=\frac{2}{3(1-\alpha)}$ & \\
\hline
\end{tabular}

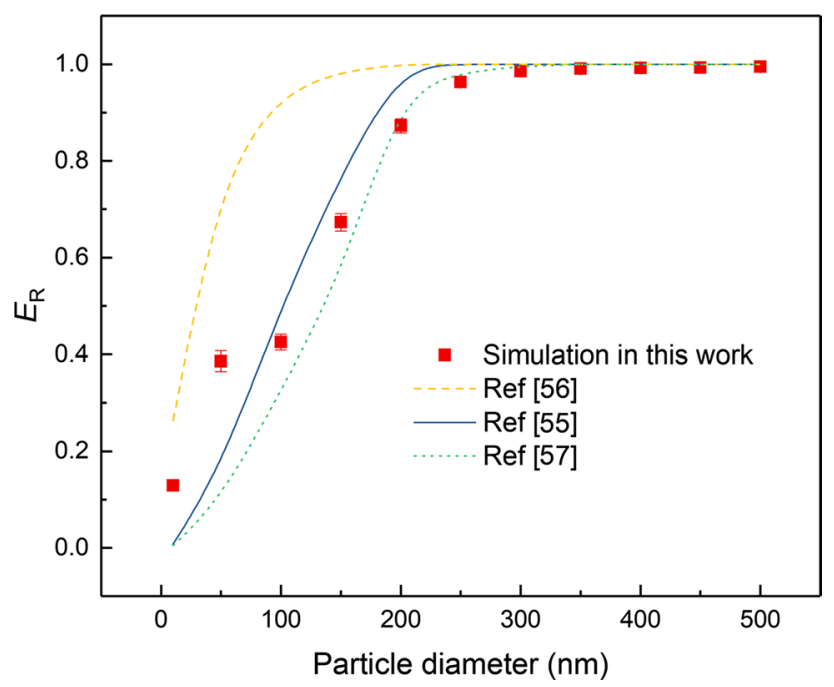

Fig. $8 E_{\mathrm{R}}$ profile simulated in this work compared with those calculated by other models (fiber diameter $=370 \mathrm{~nm} ; \mathrm{SVF}=3 \%$ )

the potential of the fiber surface was first set as 0 . With no Coulomb force considered, the simulated efficiencies in this work showed deviations smaller than those calculated by Stechkina and Fuchs [52] and Pich [56] (Fig. 9) but larger than those calculated by Liu and Rubow [53] and Lee and Gieske [57]. When the fiber potential was set as $-0.3 \mathrm{~V}$, the simulated filtration efficiencies were much closer to the experimental data, compared with those calculated by Liu and Rubow [53] and Lee and Gieske [57]. The results illustrate that the filtration efficiency for the particles of $10-289 \mathrm{~nm}$ is significantly susceptible to the modeling item of Coulomb force.

Some deviations still exist between the experimental data and the simulated values when considering the fiber potential value (Fig. 9). Fotovati et al. [48] established a series of 3D fibrous geometries with an SVF of 7.5\% and fiber diameter of $10 \mu \mathrm{m}$ to study the effect of fiber orientations on the filtration efficiency and concluded that the in-plane and the through-plane orientations of fibers had distinct effects on

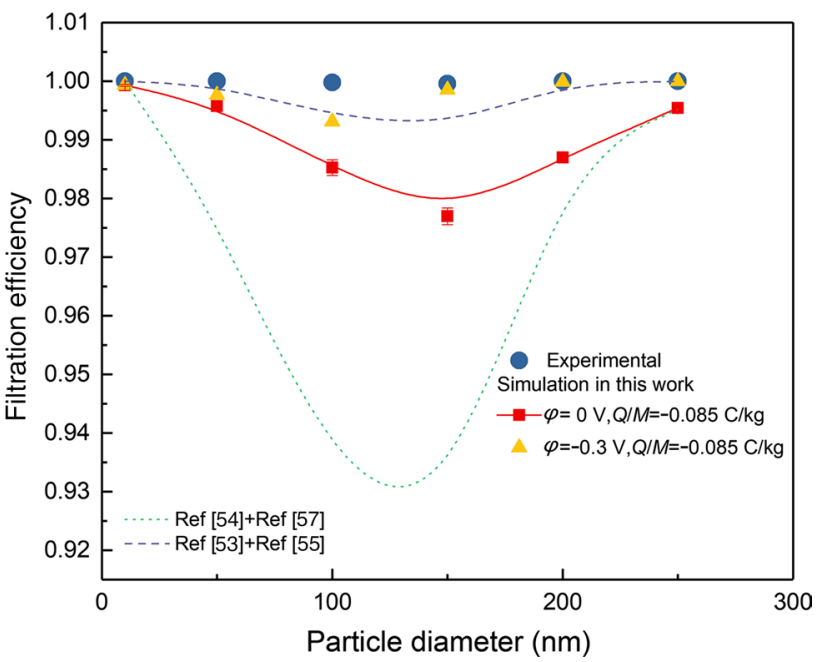

Fig. 9 Estimated filtration efficiency for aerosol particles using different models, compared with experimental data in Ref. [4]

the filtration efficiency for the particles with different sizes. In this work, we established a random fiber media model; we neglected the fibers orientation and assumed a uniform fiber potential, which is probably the major cause of the deviation.

Furthermore, using our models, we simulated the pressure drop and filtration efficiency for particles with a broad size distribution (50-500 nm), following the experimental parameters in the work of Hung and Leung [59]. Hung and Leung [59] prepared filters by electrospinning nylon- 6 nanofibers on the substrates and tested the filtration efficiency for $\mathrm{NaCl}$ aerosol particles $(50-500 \mathrm{~nm}$ at a speed of $5 \mathrm{~cm} / \mathrm{s}$ ) neutralized by an aerosol neutralizer (Po-210). The structural parameters of the filters were a mean fiber diameter of $185 \mathrm{~nm}$, fiber volume fraction of 0.0182 , and thickness of $8.4 \mu \mathrm{m}$. Their experimental data indicated that the filtration efficiency was $44.1 \%$ for the most penetrating particles of $100 \mathrm{~nm}$ and $76.2 \%$ for the $500 \mathrm{~nm}$ particles.

Figure 10 shows the estimated pressure drop and filtration efficiencies for aerosol particles $(50-500 \mathrm{~nm})$. Regarding the 


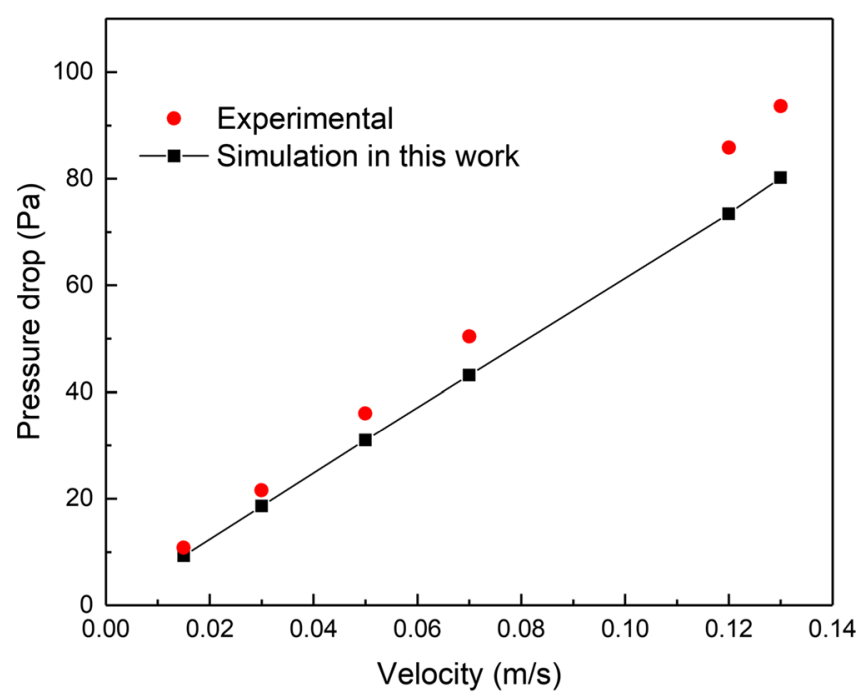

(a)

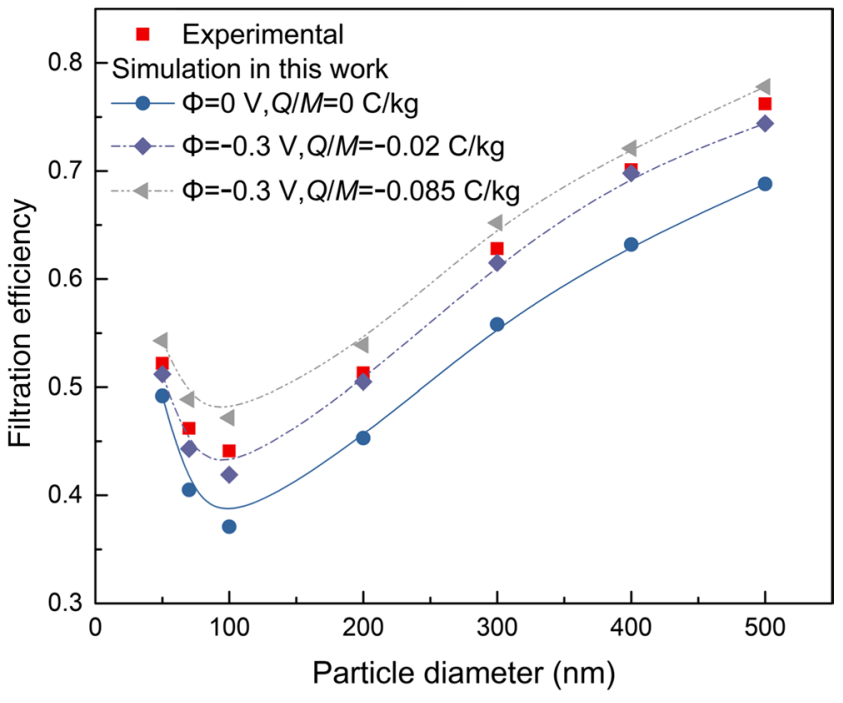

(b)

Fig. 10 (a) Estimated pressure drop and (b) filtration efficiency for aerosol particles simulated with the default value of fiber potential $(\Phi=-0.3 \mathrm{~V})$ and particle charge-to-mass ratio $(Q / M)$ versus the experimental data from Ref. [59]

pressure drop, the deviation between the simulated value and the experimental data was about $14 \%$. For example, when the velocity was $0.05 \mathrm{~m} / \mathrm{s}$, the experimental value of pressure drop was $36 \mathrm{~Pa}$ and the simulated value was $31.0 \mathrm{~Pa}$, corresponding to a deviation of $13.8 \%$ (Fig. 10a). To simulate the filtration efficiency, Coulomb force was not considered. The average deviation of filtration efficiencies for the particles (50-500 nm) versus the experimental data was about $10 \%$. In particular, for the $100 \mathrm{~nm}$ particles, the filtration efficiency was estimated as $37.1 \%$ (Fig. 10b).

Considering the effect of Coulomb forces, we again adopted a fiber potential of $-0.3 \mathrm{~V}$; however, Hung and Leung [59] did not measure the charge of their $\mathrm{NaCl}$ aerosol particles after neutralization. Tsai et al. [60] measured the charge distribution of $\mathrm{NaCl}$ particles neutralized by Po-210. They found that after $\mathrm{Po}-210$ neutralization, the $\mathrm{NaCl}$ aerosol particles $(0.25-0.70 \mu \mathrm{m})$ reached the Boltzmann charge equilibrium, with a negative charge of about $0.85-2$ elementary units of charge, while the $\mathrm{NaCl}$ particles $(0.01-0.10 \mu \mathrm{m})$ were negatively charged with $0.1-1.0$ elementary unit of charge. Thus, the particle $Q / M$ ratio was set as $-0.02 \mathrm{C} / \mathrm{kg}$ or $-0.085 \mathrm{C} / \mathrm{kg}$ in the simulation. As shown in Fig. 10b, the simulated results with a $Q / M$ value of $-0.02 \mathrm{C} / \mathrm{kg}$ provided a filtration efficiency of $41.9 \%$ for the most penetrating particles of $100 \mathrm{~nm}$, while with a $Q / M$ value of $-0.085 \mathrm{C} / \mathrm{kg}$, the estimated filtration efficiency was $47.2 \%$ for the $100 \mathrm{~nm}$ particles. The average deviations of estimated filtration efficiency were $2.5 \%$ and $4.2 \%$ at $Q / M$ values of $-0.02 \mathrm{C} / \mathrm{kg}$ and $-0.085 \mathrm{C} / \mathrm{kg}$, respectively (Fig. 10b). The results illustrate that the established models considering Coulomb forces can enhance the filtration performance simulation accuracy.

\section{Results and Discussion}

Based on the above established models considering the mechanisms of Brownian diffusion and interception capture and the Coulomb force, we studied the variation tendency of filtration efficiency and pressure drop of fibrous filters, based on factors such as fiber potential, particle $Q / M$ ratio, SVF, fiber diameter, and face velocity.

It has been reported that negatively charged aerosol particles generally have a $Q / M$ of $0.00139-0.1 \mathrm{C} / \mathrm{kg}[35,61,62]$. According to the potential feature of fibers as mentioned in the above section, we adopted $-0.3,-0.2$, and $-0.1 \mathrm{~V}$ as the potential of fiber surfaces to calculate the filtration efficiency for the $10-500 \mathrm{~nm}$ particles with different $Q / M$ ratios, ranging from -0.01 to $-0.085 \mathrm{C} / \mathrm{kg}$, maintaining the SVF value of $3 \%$ and fiber diameter of $370 \mathrm{~nm}$. Figure 11a shows that at a high fiber potential $(-0.3 \mathrm{~V})$, under a $Q / M$ value of $-0.085 \mathrm{C} / \mathrm{kg}$, the filtration efficiency increases with the particle size; the filtration efficiency is close to $10 \%$ for the $200 \mathrm{~nm}$ particles, while it approximates $70 \%$ for the $500 \mathrm{~nm}$ particles. When the fiber potential is lower $(-0.2$ and $-0.1 \mathrm{~V}$ ), the filtration efficiency tends to increase with the particle size, but the value decreases to some extent. Under lower $Q / M$ values $(-0.01,-0.02,-0.05 \mathrm{C} / \mathrm{kg})$, the filtration efficiency reduces greatly, although it varies similarly with the particle size. As shown in Fig. S4, for the $500 \mathrm{~nm}$ particles, the filtration efficiency is about $46.40 \%$ at $Q / M$ value of $-0.05 \mathrm{C} / \mathrm{kg}$, whereas it decreases to $12.06 \%$ at $Q / M$ value of $-0.01 \mathrm{C} / \mathrm{kg}$, even when the fiber potential is maintained at $-0.3 \mathrm{~V}$. Figure $11 \mathrm{~b}$ shows the filtration efficiency for the $300 \mathrm{~nm}$ particles. The filtration efficiency increases with 


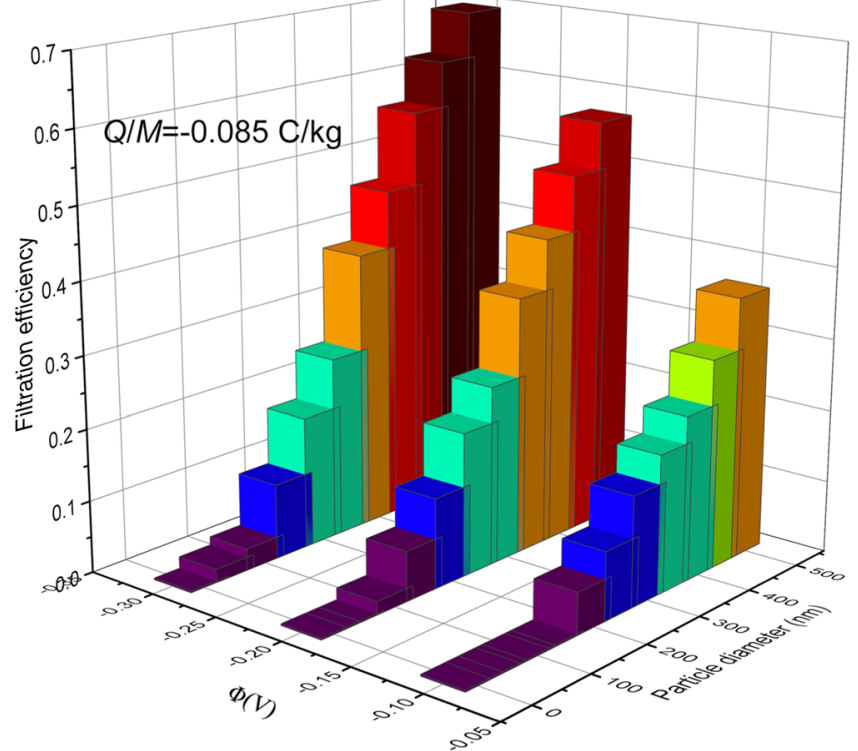

(a)

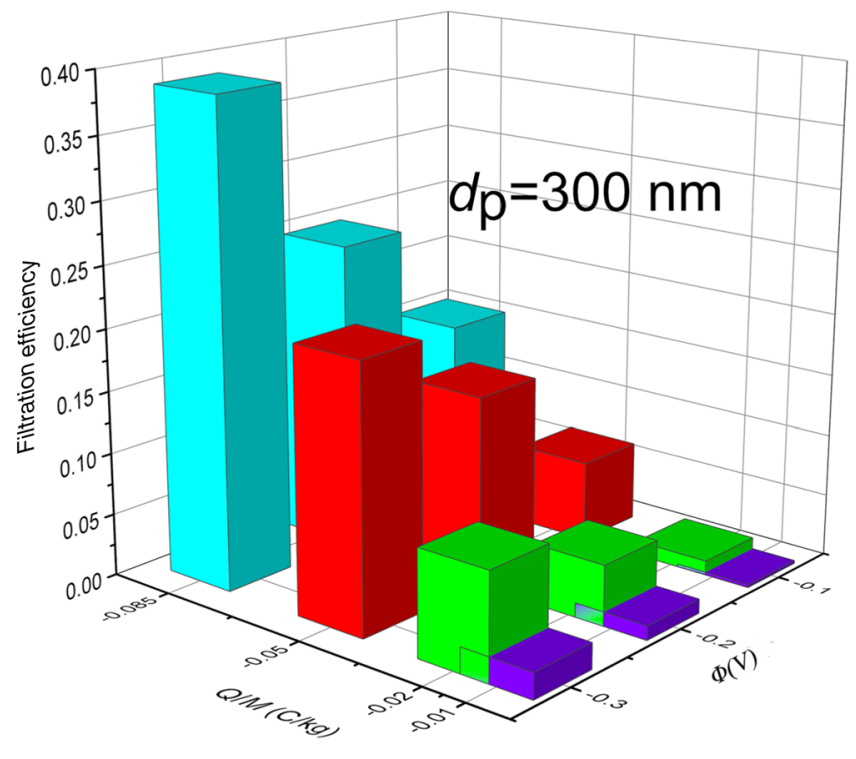

(b)

Fig. 11 Filtration efficiency for aerosol particles (with $Q / M$ value of $-0.085 \mathrm{C} / \mathrm{kg}$ ) as a function of fiber potential $(\Phi)$ and particle size $(\mathbf{a})$ and for the $300 \mathrm{~nm}$ particles, related to the particle charge-to-mass ratio $(Q / M)$ and the fiber potential $(\mathbf{b})$

the absolute values of $Q / M$ and the fiber potential. Givehchi et al. [63] found that when the particle size was increased from $10 \mathrm{~nm}$ to $100 \mathrm{~nm}$, the efficiency caused by Coulomb force increased from almost negligible to $30 \%$. According to the study by Wang [26], particle trapping caused by Coulomb force increased as the particle charge and fiber potential increased.

Furthermore, the effects of the SVF on the filtration efficiency and the pressure drop were studied with a fiber diameter of $370 \mathrm{~nm}$, fiber potential of $-0.3 \mathrm{~V}$, and particle $Q / M$ ratio of $-0.085 \mathrm{C} / \mathrm{kg}$. As shown in Fig. 12, the pressure drop increased almost linearly with the SVF, which is due to the positive correlation expressed by Eqs. (7) and (8). However, the filtration efficiency for the particles showed no significant increase at a high SVF value. For the $400 \mathrm{~nm}$ particles, the filtration efficiency was about $99.871 \%$ at an SVF of $2 \%$ and $99.991 \%$ at an SVF of $3 \%$. It exhibited a similar tendency for the $300 \mathrm{~nm}$ particles. In the study by Yun et al. [64], when the fiber diameter of a polyacrylonitrile filter was fixed at $270 \mathrm{~nm}$, as the SVF increased from 11.2 to $15.2 \%$, the pressure drop increased from 10062 to $14750 \mathrm{~Pa} / \mathrm{mm}$, and the filtration efficiency also increased. $\mathrm{Li}$ et al. [65] simulated eight flow fields of filter media with different SVFs and found that the pressure drop increased nonlinearly with the SVF. Soltani et al. [66] studied the effect of 3D fiber SVF on permeability and concluded that SVFs were positively correlated with filtration efficiency. Hosseini and Tafreshi [21] studied the effects of six different fiber diameters (100-1000 nm) on filtration efficiency under three

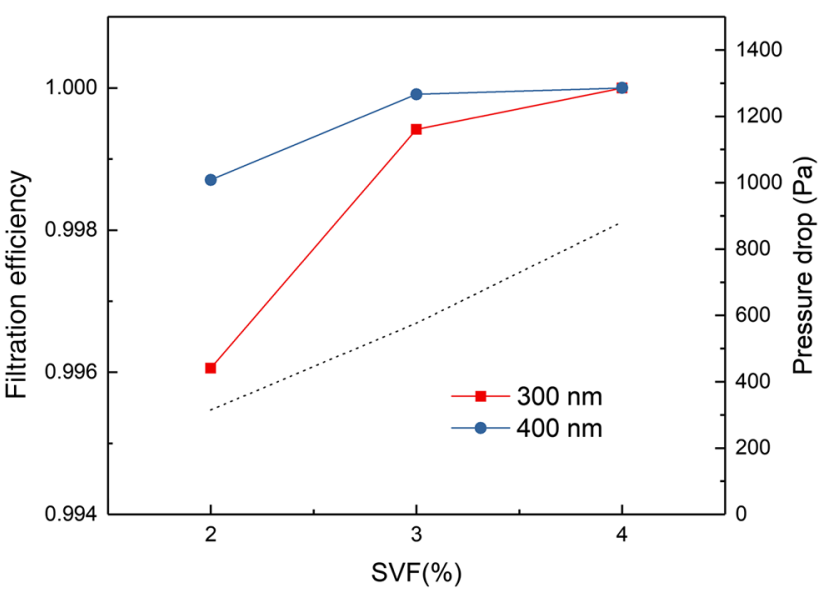

Fig. 12 Filtration efficiency (solid line) and pressure drop (dash line) for $300 \mathrm{~nm}$ and $400 \mathrm{~nm}$ particles as a function of solid volume fraction (SVF)

different SVFs $(2.5 \%, 5 \%, 7.5 \%)$. Our simulated results are consistent with these tendencies in the literature.

The effects of fiber diameter on the filtration efficiency and pressure drop were studied with an SVF of $3 \%$, fiber potential of $-0.3 \mathrm{~V}$, and $Q / M$ of $-0.085 \mathrm{C} / \mathrm{kg}$. As shown in Fig. 13, under the same SVF value, the pressure drop decreased as the fiber diameter rose from 200 to $500 \mathrm{~nm}$, and the pressure drop value was independent of particle size. When the fiber diameter was $500 \mathrm{~nm}$, the filtration efficiency decreased with an increase in the particle size 


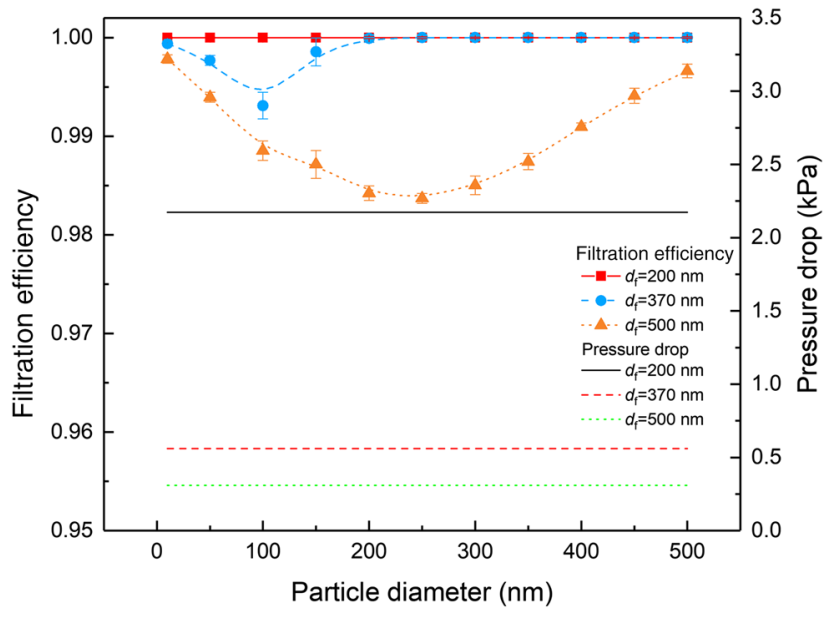

Fig. 13 Filtration efficiency and pressure drop as a function of particle size $(10-500 \mathrm{~nm})$, based on the fiber diameters

from 50 to $250 \mathrm{~nm}$ and then increased with an increase in the particle size from 250 to $500 \mathrm{~nm}$, achieving a filtration efficiency of $99.66 \%$ for the $500 \mathrm{~nm}$ particles and the minimum value of $98.37 \%$ for the $250 \mathrm{~nm}$ particles. For the case of thin fiber diameter $(370 \mathrm{~nm})$, the filtration efficiency had a minimum value of $99.31 \%$ for certain particles around $100 \mathrm{~nm}$ and then reached about $100 \%$ for the particles larger than $200 \mathrm{~nm}$. When the fiber diameter was decreased to $200 \mathrm{~nm}$, the filtration efficiency was about $100 \%$ for all the particles $(10-500 \mathrm{~nm})$. When the fiber diameter was $200 \mathrm{~nm}, 370 \mathrm{~nm}$, and $500 \mathrm{~nm}$, the most penetrating particle sizes (MPPSs) were $50 \mathrm{~nm}, 100 \mathrm{~nm}$, and $250 \mathrm{~nm}$, respectively. Previous works have reported a similar relationship between the fiber diameter and the MPPS. Podgórski et al. [67] found that when the fiber diameter was increased from $100 \mathrm{~nm}$ to $10 \mu \mathrm{m}$, the MPPS increased from $54 \mathrm{~nm}$ to $366 \mathrm{~nm}$. Balgis et al. [68] used monodisperse aerosol particles with a diameter of $100 \mathrm{~nm}$ to perform a particle penetration test and pressure drop measurement through an air filter composed of fibers of different diameters and found that as the fiber diameter increased, the filtration efficiency decreased but the pressure drop increased.

The effects of face velocity on the pressure drop and the filtration efficiency for the $300 \mathrm{~nm}$ particles were investigated with an SVF of 3\%, fiber potential of $-0.3 \mathrm{~V}$, and $Q / M$ of $-0.085 \mathrm{C} / \mathrm{kg}$, adopting different fiber diameters. As shown in Fig. 14, the pressure drop increased with the velocity, while the relationship between the filtration efficiency and the velocity was significantly associated with the fiber diameter. With a large fiber diameter $(500 \mathrm{~nm})$, the filtration efficiency decreased from $98.95 \%$ at the velocity of $0.05 \mathrm{~m} / \mathrm{s}$ to $91.17 \%$ at $0.45 \mathrm{~m} / \mathrm{s}$. When the fiber diameter was reduced to $370 \mathrm{~nm}$, the filtration efficiency was $98.14 \%$ at $0.45 \mathrm{~m} / \mathrm{s}$. For a much thinner

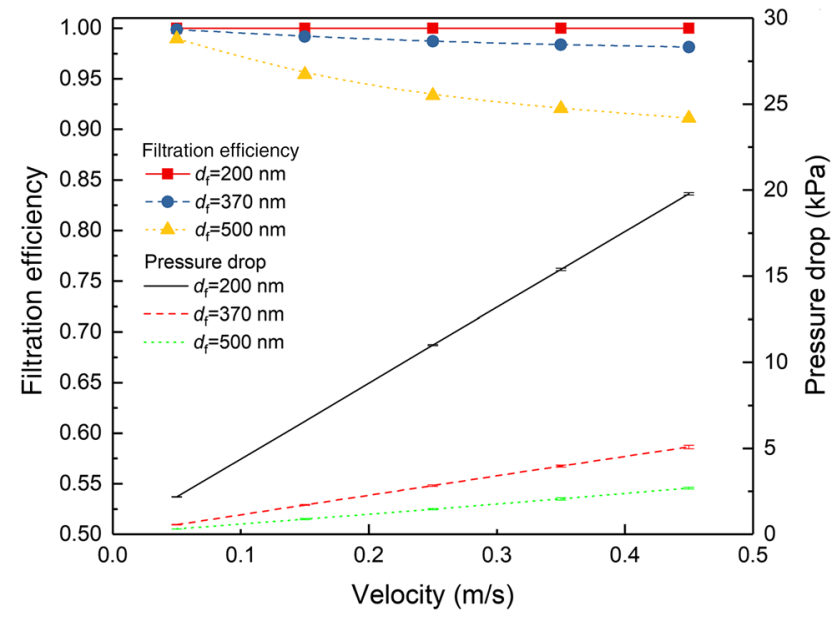

Fig. 14 Filtration efficiency and pressure drop for the $300 \mathrm{~nm}$ particles as a function of the face velocity, based on the fiber diameter

fiber $(200 \mathrm{~nm})$, at $0.45 \mathrm{~m} / \mathrm{s}$ the filtration efficiency was $99.99 \%$. This indicates that the thinner the filter fiber, the higher the filtration efficiency. Similarly, it has been reported that when the velocity increased from $0.05 \mathrm{~m} / \mathrm{s}$ to $0.1 \mathrm{~m} / \mathrm{s}$, the filtration efficiency of a commercial HEPA filter for $300 \mathrm{~nm}$ particles reduced from 99.99 to $99.98 \%$ and the pressure drop increased from 250 to $500 \mathrm{~Pa}$ [69].

\section{Conclusion}

In this study, computational models were established and verified to determine the quantitative effects of Coulomb forces on the filtration efficiency of aerosol particles, while considering the interception capture and Brownian diffusion. First, a software-coupling method was developed by interconnecting MATLAB, AutoCAD, ICEM, and Fluent software to generate a $3 \mathrm{D}$ random fiber model to describe the microstructure of fibrous filters. Then, computational models including the flow model, particle model, and electric field model were developed using the Fluent custom UDF program, assuming a uniform fiber potential and neglecting the non-uniformity of particle charge distribution. The simulated results of the pressure drop and the filtration efficiency were compared with those estimated using modeling equations in the literature. The Coulomb force was found to be the key factor required to estimate the filtration efficiency of ultrafine particles.

Furthermore, using the established computational models, the variation tendencies of the filtration efficiency and the pressure drop of fibrous filters were studied based on the influence factors of the fiber potential, particle $Q / M$ ratio, SVF, fiber diameter, and face velocity. By comparing with the experimental data in the literature, the filtration efficiency was found to increase with the particle size; 
however, the value is greatly associated with the particle $Q / M$ ratio. The pressure drop increased almost linearly with the SVF, while the filtration efficiency for the particles exhibited no significant increase at a high SVF. The filtration efficiency became larger as the fiber diameter reduced. The established models and the estimated results will provide important guidance on the design of HEPA filters for aerosol particles.

Acknowledgements This work was supported by the National Key Research and Development Program (No. 2016YFC1201503), Natural Science Foundation of China (No. 21576206) and the Program for Changjiang Scholars and Innovative Research Team in University (No. IRT_15R46).

Open Access This article is distributed under the terms of the Creative Commons Attribution 4.0 International License (http://creativeco mmons.org/licenses/by/4.0/), which permits unrestricted use, distribution, and reproduction in any medium, provided you give appropriate credit to the original author(s) and the source, provide a link to the Creative Commons license, and indicate if changes were made.

\section{References}

1. Zhao X, Li Y, Hua T et al (2017) Low-resistance dual-purpose air filter releasing negative ions and effectively capturing PM2.5. ACS Appl Mater Interface 9(13):12054-12063

2. Al-Attabi R, Dumée LF, Kong L et al (2018) High efficiency poly(acrylonitrile) electrospun nanofiber membranes for airborne nanomaterials filtration. Adv Eng Mater 20(1):1700572

3. Singh VK, Ravi SK, Sun WX et al (2017) Transparent nanofibrous mesh self-assembled from molecular LEGOs for high efficiency air filtration with new functionalities. Small 13(6):1601924

4. Bortolassi ACC, Guerra VG, Aguiar ML (2017) Characterization and evaluate the efficiency of different filter media in removing nanoparticles. Sep Purif Technol 175:79-86

5. Van Osdell DW, Liu BYH, Rubow KL et al (1990) Experimental study of submicrometer and ultrafine particle penetration and pressure drop for high efficiency filters. Aerosol Sci Technol 12(4):911-925

6. Li J, Leavey A, Wang Y et al (2018) Comparing the performance of 3 bioaerosol samplers for influenza virus. J Aerosol Sci 115:133-145

7. Thorne PS, Reynolds SJ, Milton DK et al (1997) Field evaluation of endotoxin air sampling assay methods. Am Ind Hyg Assoc J 58(11):792-799

8. Harstad JB (1965) Sampling submicron T1 bacteriophage aerosols. Appl Environ Microbiol 13(6):899-908

9. Yuan QP, Zhang H, Qian ZM et al (2004) Pilot-plant production of xylo-oligosaccharides from corncob by steaming, enzymatic hydrolysis and nanofiltration. J Chem Technol Biotechnol 79(10): 1073-1079

10. Yuan JS, Ji ZY, Chen JX et al (2013) Resource utilization of concentrated seawater generated during desalination. Hebei Univ Technol 42(1):29-35

11. Yeom BY, Shim E, Pourdeyhimi B (2010) Boehmite nanoparticles incorporated electrospun nylon-6 nanofiber web for new electret filter media. Macromol Res 18(9):884-890
12. Tang M, Thompson D, Chang DQ et al (2018) Filtration efficiency and loading characteristics of PM 2.5 through commercial electret filter media. Sep Purif Technol 195:101-109

13. Zhu M, Han J, Wang F et al (2017) Electrospun nanofibers membranes for effective air filtration. Macromol Mater Eng 302(1): 1600353

14. Gervais PC, Bourrous S, Dany F et al (2015) Simulations of filter media performances from microtomography-based computational domain. Experimental and analytical comparison. Comput Fluids 116:118-128

15. Qin XH, Wang SY (2006) Filtration properties of electrospinning nanofibers. J Appl Polym Sci 102(2):1285-1290

16. Huang $H$, Zheng $C$, Zhao $H$ (2017) Numerical investigation on non-steady-state filtration of elliptical fibers for submicron particles in the "Greenfield gap" range. J Aerosol Sci 114:263-275

17. Payatakes AC, Gradoń L (1980) Dendritic deposition of aerosol particles in fibrous media by inertial impaction and interception. Chem Eng Sci 35(5):1083-1096

18. Ramarao BV, Chi TE, Mohan S (1994) Calculation of single fiber efficiencies for interception and impaction with superposed Brownian motion. J Aerosol Sci 25(2):295-313

19. Zhu C, Lin $\mathrm{CH}$, Cheung CS (2000) Inertial impaction-dominated fibrous filtration with rectangular or cylindrical fibers. Powder Technol 112(1):149-162

20. Wang H, Zhao H, Wang K et al (2014) Simulating and modeling particulate removal processes by elliptical fibers. Aerosol Sci Technol 48(2):207-218

21. Hosseini SA, Tafreshi HV (2010) 3-D simulation of particle filtration in electrospun nanofibrous filters. Powder Technol 201(2):153-160

22. Hosseini SA, Tafreshi HV (2012) Modeling particle-loaded single fiber efficiency and fiber drag using ANSYS-Fluent CFD code. Comput Fluids 66:157-166

23. Huang B, Yao Q, Li SQ et al (2006) Experimental investigation on the particle capture by a single fiber using microscopic image technique. Powder Technol 163(3):125-133

24. Cai RR, Zhang LZ (2016) Modeling of dynamic deposition and filtration processes of airborne particles by a single fiber with a coupled lattice Boltzmann and discrete element method. Build Environ 106:274-285

25. Wang H, Zhao H, Wang K et al (2013) Simulation of filtration process for multi-fiber filter using the Lattice-Boltzmann twophase flow model. J Aerosol Sci 66:164-178

26. Wang CS (2001) Electrostatic forces in fibrous filters-a review. Powder Technol 118(1-2):166-170

27. Shou D, Fan J, Zhang H et al (2015) Filtration efficiency of nonuniform fibrous filters. Aerosol Sci Technol 49(10):912-919

28. Babaie M, Talebi S, Abouali O (2018) Numerical investigation on dust-loaded fibrous filters. J Braz Soc Mech Sci 40(4):223

29. Kuwabara S (1959) The forces experienced by randomly distributed parallel circular cylinders or spheres in a viscous flow at small Reynolds numbers. J Phys Soc Jpn 14(4):527-532

30. Dong M, Li J, Shang Y et al (2019) Numerical investigation on deposition process of submicron particles in collision with a single cylindrical fiber. J Aerosol Sci 129:1-15

31. Gopan A, Yang Z, Axelbaum RL (2019) Predicting particle deposition for flow over a circular cylinder in combustion environments. Proc Combust Inst 37(4):4427-4434

32. Hosseini SA, Tafreshi HV (2010) Modeling particle filtration in disordered 2-D domains: a comparison with cell models. Sep Purif Technol 74(2):160-169

33. Jin X, Yang L, Du X (2017) Modeling filtration performance of elliptical fibers with random distributions. Adv Powder Technol 28(4):1193-1201 
34. Nielsen KA, Hill JC (1976) Capture of particles on spheres by inertial and electrical forces. Ind Eng Chem Fundam 15(3): 157-163

35. D'Addio L, di Natale F, Carotenuto C et al (2013) A lab-scale system to study submicron particles removal in wet electrostatic scrubbers. Chem Eng Sci 97:176-185

36. Hamaguchi S, Farouki RT (1994) Polarization force on a charged particulate in a nonuniform plasma. Phys Rev E 49(5):4430

37. Kanaoka C, Hiragi S, Tanthapanichakoon W (2001) Stochastic simulation of the agglomerative deposition process of aerosol particles on an electret fiber. Powder Technol 118(1-2):97-106

38. Zuo Z, Wang J, Huo Y et al (2016) Particle motion induced by electrostatic force of a charged droplet. Environ Eng Sci 33(9):650-658

39. Adamiak K (1995) Aerosol deposition on an arbitrarily oriented single rectangular fibre in a uniform electric field. In: IAS'95. conference record of the 1995 IEEE industry applications conference thirtieth IAS annual meeting. IEEE, vol. 2, pp. 1385-1389

40. Hoppel WA (1976) Ion-aerosol attachment coefficients and the diffusional charging of aerosols. Electrical processes in atmospheres. Springer, Heidelberg, pp 60-69

41. Hoppel WA (1985) Ion-aerosol attachment coefficients, ion depletion, and the charge distribution on aerosols. J Geophys ResAtmos 90(D4):5917-5923

42. Dhanorkar S, Kamra AK (2001) Effect of coagulation on the particle charge distribution and air conductivity. J Geophys Res-Atmos 106(D11):12055-12065

43. Saleh AM, Tafreshi HV, Pourdeyhimi B (2016) An analytical approach to predict pressure drop and collection efficiency of dust-load pleated filters. Sep Purif Technol 161:80-87

44. Huang H, Wang K, Zhao H (2016) Numerical study of pressure drop and diffusional collection efficiency of several typical noncircular fibers in filtration. Powder Technol 292:232-241

45. Davies C (1973) Air filtration. Academic Press, London

46. Happel J (1959) Viscous flow relative to arrays of cylinders. AIChE J 5(2):174-177

47. Tek MR (1957) Development of a generalized Darcy equation. J Pet Technol 9(6):45-47

48. Fotovati S, Vahedi Tafreshi H, Pourdeyhimi B (2010) Influence of fiber orientation distribution on performance of aerosol filtration media. Chem Eng Sci 65(18):5285-5293

49. Hinds WC (2012) Aerosol technology: properties, behavior, and measurement of airborne particles. Wiley, Hoboken

50. Brown RC (1984) A many-fibre model of airflow through a fibrous filter. J Aerosol Sci 15(5):583-593

51. Lee KW, Liu BYH (1982) Theoretical study of aerosol filtration by fibrous filters. Aerosol Sci Technol 1(2):147-161

52. Stechkina IB, Fuchs NA (1966) Studies on fibrous aerosol filtersI. Calculation of diffusional deposition of aerosols in fibrous filters. Ann Occup Hyg 9(2):59-64
53. Liu BYH, Rubow KL (1990) Efficiency, pressure drop and figure of merit of high efficiency fibrous and membrane filter media. In: Proceedings of the fifth world filtration congress 9

54. Payet S, Boulaud D, Madelaine G et al (1992) Penetration and pressure drop of a HEPA filter during loading with submicron liquid particles. JAerosol Sci 23(7):723-735

55. Lee KW, Liu BYH (1982) Experimental study of aerosol filtration by fibrous filters. Aerosol Sci Technol 1(1):35-46

56. Pich J (1966) The effectiveness of the barrier effect in fiber filters at small Knudsen numbers. Staub Reinhalt Luft 26:1-4

57. Lee KW, Gieseke JA (1980) Note on the approximation of interceptional collection efficiencies. J Aerosol Sci 11(4):335-341

58. Oyama Y, Osaki T, Kamiya K et al (2012) A glass fiber sheetbased electroosmotic lateral flow immunoassay for point-of-care testing. Lab Chip 12(24):5155-5159

59. Hung CH, Leung WWF (2011) Filtration of nano-aerosol using nanofiber filter under low Peclet number and transitional flow regime. Sep Purif Technol 79(1):34-42

60. Tsai CJ, Lin JS, Deshpande CG et al (2005) Electrostatic charge measurement and charge neutralization of fine aerosol particles during the generation process. Part Part Syst Charact 22(5):293-298

61. Wong J, Lin YW, Kwok PCL et al (2015) Measuring bipolar charge and mass distributions of powder aerosols by a novel tool (BOLAR). Mol Pharm 12(9):3433-3440

62. Mazumder MK, Sims RA, Biris AS et al (2006) Twenty-first century research needs in electrostatic processes applied to industry and medicine. Chem Eng Sci 61(7):2192-2211

63. Givehchi R, Li Q, Tan Z (2015) The effect of electrostatic forces on filtration efficiency of granular filters. Powder Technol 277:135-140

64. Yun KM, Jr HoganCJ, Matsubayashi Y et al (2007) Nanoparticle filtration by electrospun polymer fibers. Chem Eng Sci 62(17):4751-4759

65. Li YY, Fu HM, Hu YL (2011) Numerical simulation of three dimensional flow fields of fiber filter media. J Text Res 32(5):16-21

66. Soltani P, Johari MS, Zarrebini M (2014) Effect of 3D fiber orientation on permeability of realistic fibrous porous networks. Powder Technol 254:44-56

67. Podgórski A, Bałazy A, Gradoń L (2006) Application of nanofibers to improve the filtration efficiency of the most penetrating aerosol particles in fibrous filters. Chem Eng Sci 61(20):6804-6815

68. Balgis R, Kartikowati CW, Ogi T et al (2015) Synthesis and evaluation of straight and bead-free nanofibers for improved aerosol filtration. Chem Eng Sci 137:947-954

69. Ahn YC, Park SK, Kim GT et al (2006) Development of high efficiency nanofilters made of nanofibers. Curr Appl Phys 6(6):1030-1035 